\title{
Image of the month: All puffed out - a case of crack lung
}

\author{
Authors: Aryeh Greenberg, ${ }^{A}$ Kathryn Stammers, ${ }^{B}$ Ian Moonsie ${ }^{C}$ and Ricardo J José ${ }^{D}$
}

A previously well 33-year-old man presented to the emergency department with a 48-hour history of worsening shortness of breath, dry cough, chest pain and fevers. He had no haemoptysis and reported no recent travel abroad. He had ordinarily smoked crack cocaine for the past 20 years although, in the 3 days preceding presentation to hospital, he had increased his cocaine use considerably. Peripheral blood oxygen saturation $\left(\mathrm{SpO}_{2}\right)$ was $90 \%$ on room air and he was notably tachypnoeic. On examination, he exhibited no finger clubbing, peripheral stigmata of endocarditis or cervical lymphadenopathy. Bibasal crepitations were audible on praecordial auscultation. Blood panels showed mild neutrophilia of $10.19 \times 10^{9} / \mathrm{L}$, normal eosinophil count, normal C-reactive protein and normocytic anaemia (haemoglobin $112 \mathrm{~g} / \mathrm{L}$ ). Haematinics were within normal limits and he was negative for HIV. Plain chest radiography (Fig 1) showed bilateral upper and mid-zone opacification with right lower zone and left mid-zone consolidation, but no effusions or collapse. Computerised tomography with contrast (Fig 2) demonstrated extensive bilateral ground-glass opacities in an alveolar distribution, with air bronchograms throughout. These changes spared the peripheral lung parenchyma. No mediastinal or axillary lymphadenopathy was in evidence. The patient was initially started on empirical antibiotics to cover the possibility of atypical pneumonia. In view of the above findings, a diagnosis of acute alveolitis secondary to crack cocaine inhalation was made. Although unlikely in our case, the main differentials were acute respiratory distress syndrome, heart failure and pneumonia, including Pneumocystis jirovecii pneumonia and acute eosinophilic pneumonia.

At follow-up 1 week later, and having abstained from cocaine smoking in the interim, the patient reported a dramatic improvement in his breathing, back to premorbid baseline. $\mathrm{SpO}_{2}$

Authors: ${ }^{\text {A }}$ core medical trainee year 1 , North Middlesex University Hospital NHS Trust, London, UK; ${ }^{\text {B }}$ foundation year 2 doctor, North Middlesex University Hospital NHS Trust, London, UK; C ${ }^{C}$, respiratory physician, North Middlesex University Hospital NHS Trust, London, UK; ${ }^{\mathrm{D}}$ academic clinical lecturer in translational medicine, University College London, London, UK and ST6 in respiratory medicine, North Middlesex University Hospital NHS Trust, London, UK

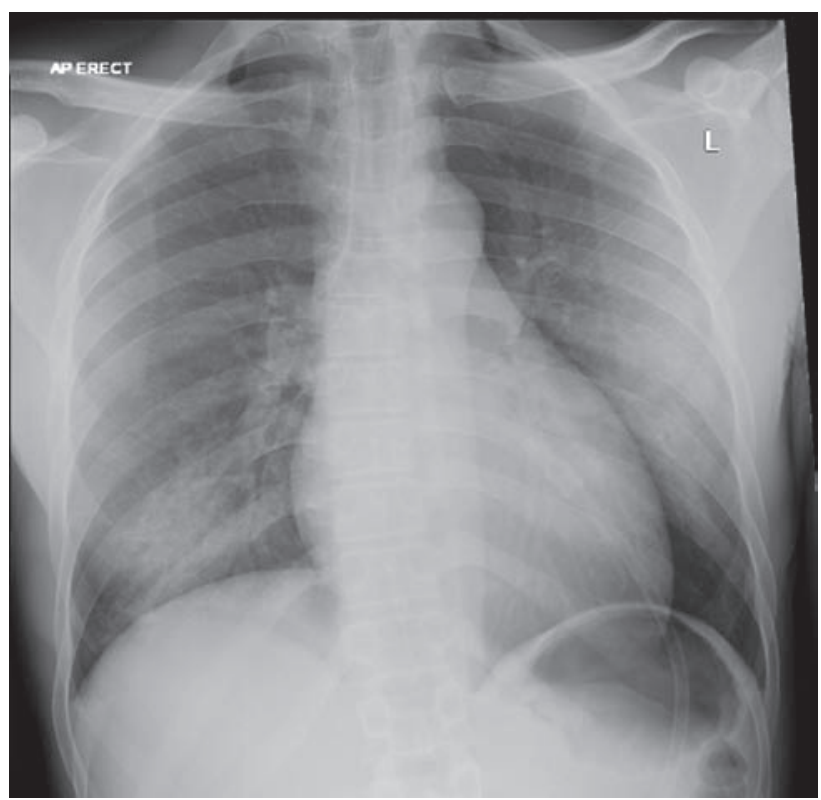

Fig 1. Chest radiograph on admission, showing bilateral upper and mid-zone opacification with right lower zone and left mid-zone consolidation.

was $99 \%$ on air. A repeat chest radiograph (Fig 3) illustrated complete resolution.

Clinico-radiological correlation - in particular, the temporal relationship between cocaine use and symptom onset - clinched the diagnosis as 'crack lung', otherwise called cocaine-induced lung injury, a syndrome of diffuse alveolar damage and haemorrhagic alveolitis occurring within 48 hours of smoking crack cocaine. ${ }^{1}$ The correct diagnosis was reached by paying close attention to the drug history and confirmed retrospectively by observing complete symptomatic and radiological resolution upon smoking cessation.

\section{Conflicts of interest}

The authors have no conflicts of interest to declare.

\section{Author contributions}

AG drafted the manuscript, KS reviewed the patient in clinic, RJJ and IM advised on the case and critically appraised the draft manuscript. 


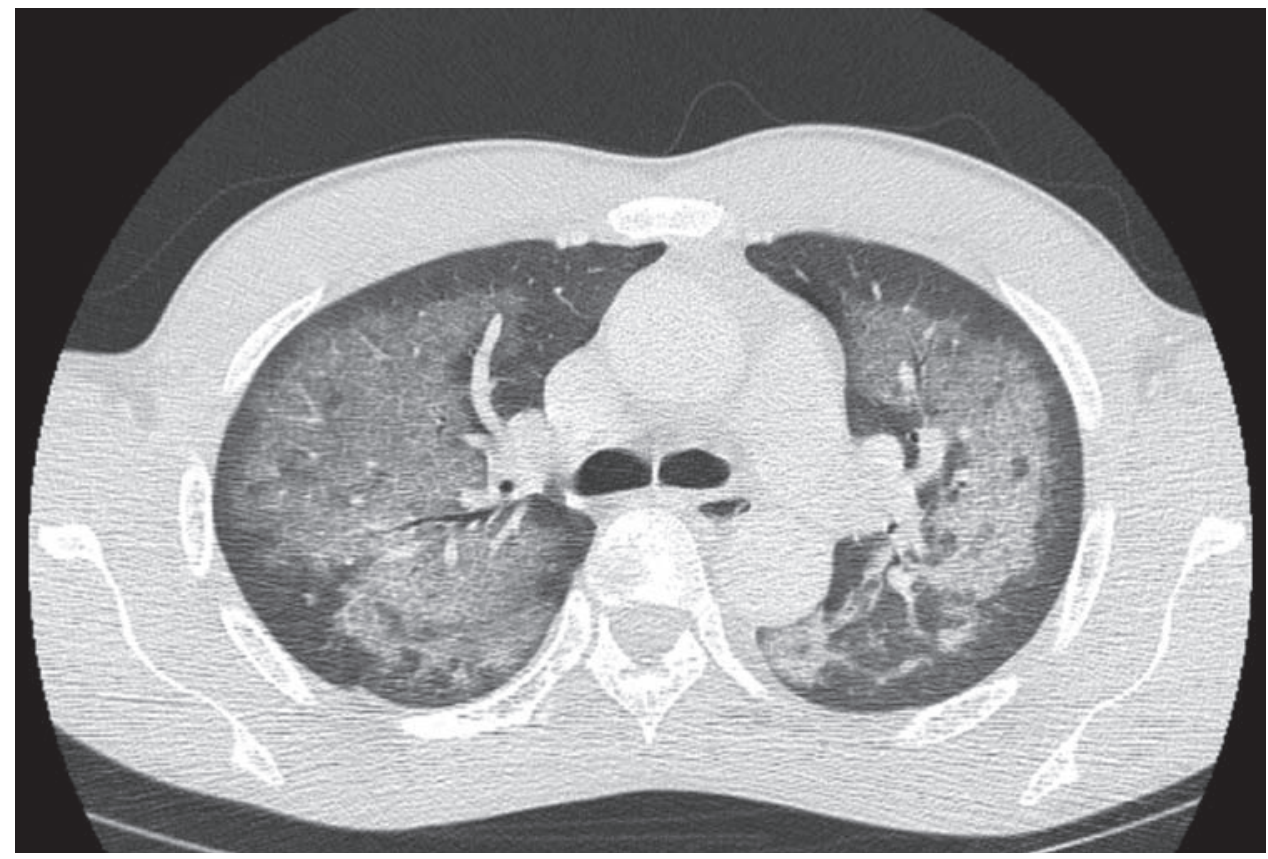

Fig 2. Computerised tomography chest with contrast demonstrating, at the level of the carina, extensive bilateral ground-glass opacities with air bronchograms in an alveolar distribution sparing the periphery.

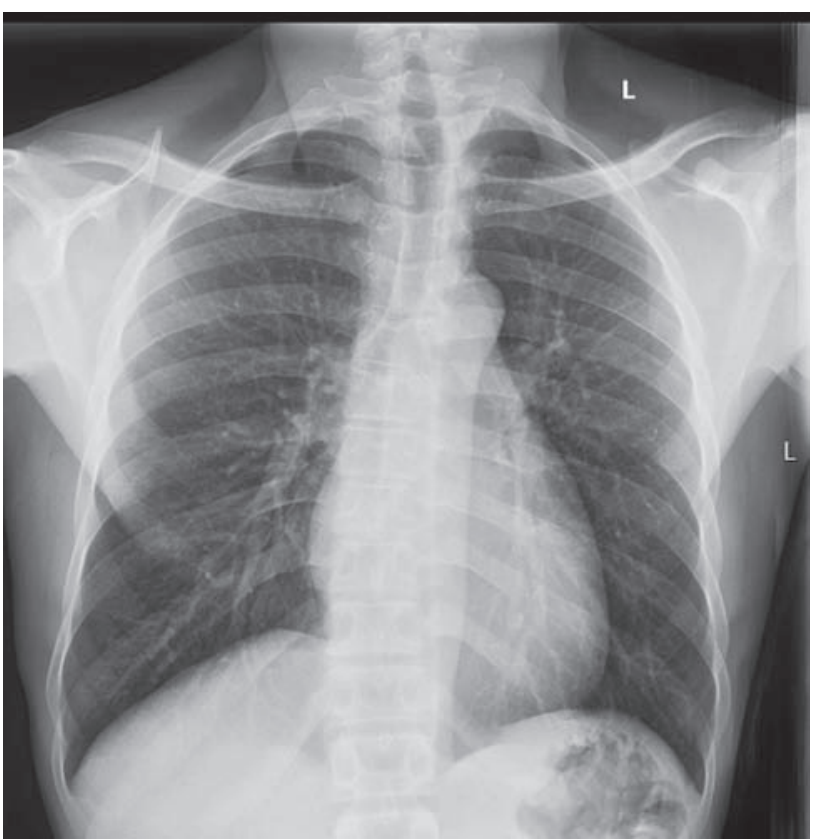

\section{Acknowledgements}

Consent was obtained from the patient for publication of the clinical details and images in this article.

\section{Reference}

1 Zimmerman JL. Cocaine intoxication. Crit Care Clin 2012;28:517-26.

Address for correspondence: Dr Aryeh Greenberg, North Middlesex University Hospital NHS Trust, Sterling Way, London N18 1QX, UK.

Email: aryeh.greenberg@nhs.net

Fig 3. A repeat chest radiograph 7 days following presentation illustrated complete radiological resolution. 